\section{Anleitung zur Ohrpräparation}

D urch die Initiave von Frau Dr. Hildmann konnte nach dem frühen Tod von Prof. Henning Hildmann unter Mitwirkung des Kliniknachfolgers Prof. Dr. Stefan Dazert und seines Schülers Prof. Dr. Holger Sudhoff ein unvollendetes Werk fertiggestellt werden. Unter dem Titel „Manual of temporal bone exercise" liegt eine faszinierende Anleitung zur Ohrpräparation vor.

Das Buch ist in englischer Sprache geschrieben und richtet sich natürlicherweise zunächst an alle, die einen Einstieg in die Ohrchirurgie suchen. Aber nicht nur "Einsteiger" sondern auch "alte Hasen“ kommen in dieser vorzüglich bebilderten und illustrierten Aus- gabe auf ihre Kosten. Die Grundlage bildet natürlich die Plestersche Ohrchirurgielehre.

Das Werk ist mit fast 40 Seiten erstaunlich kurz gehalten aber dennoch reich an Inhalt. Die Bilder und Illustrationen sind von einer Qualität, die auch den gehobenen modernen Ansprüchen genügt.

Mit dem Werk ist zu guter Letzt auch noch eine gute Tat verbunden. Die Autoren erklären, dass alle Erlöse aus diesem Buch dem gemeinsamen Ruanda-Projekt des Berufsverbandes der Deutschen HNO-Ärzte und der Deutschen Gesellschaft für Hals-Nasen-Ohren-Heilkunde, Kopf- und Hals-chirurgie zugute kommen sollen. In diesem Projekt werden

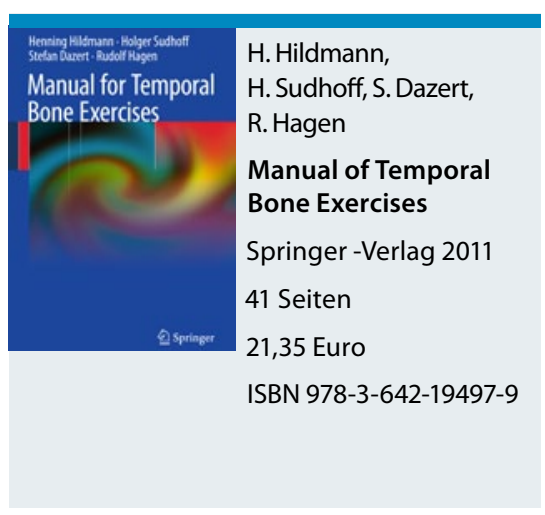

junge Assistenzärzte ganz im Sinne von Henning Hildmann und ausgehend von seiner Initiative im eigenen Land $\mathrm{zu}$ HNO-Ärzten durch erfahrene deutsche Kollegen aus Klinik und Praxis weitergebildet.

Dr. med. Joachim Wichmann

\title{
Von Kopf bis Hals durchleuchtet
}

$\mathrm{D}$ as aufwendig gestaltete Buch zur Kopf-Hals-Radiologie vermittelt auf den ersten Blick einen soliden Einstieg in diese komplexe Thematik. Die Kapitel zu den einzelnen Regionen des Kopf-Hals-Bereiches sind einheitlich aufgebaut: Zunächst wird die normale Anatomie dargestellt, anschließend die unterschiedlichen Krankheitsbilder, wie z.B. angeborene Veränderungen, Entzündungen, Tumoren sowie traumatische bzw. vaskuläre Veränderungen. Über die „klassischen“ HNOGebiete hinaus werden - sehr wichtig für differenzialdiagnostische Überlegungen bei Erkrankungen in den Nachbarschaftsregionen unseres $\mathrm{Fa}$ ches - auch Orbita, Kiefergelenk, Zahn und Kiefer sowie die Schilddrüse abgehandelt.

Leider sind die einzelnen Kapitel von sehr unterschiedlicher Qualität - einer der Nachteile, wenn zu viele „Köche“ an einem Buch beteiligt sind. Das fällt vor allem bei der Darstellung der normalen Anatomie auf, ohne deren Kenntnis die Interpretation pathologischer Befunde kaum möglich ist; im Beitrag zum Felsenbein beispielsweise werden die radiologischen Grundlagen ausführlich und mit sorgfältig ausgesuchtem und beschriftetem Bildmaterial vorgestellt, während im Nasennebenhöhlen-Kapitel für die Erklärung der Normalanatomie gerade einmal drei Schnittbilder (je ein koronares und axiale CT-Bild und ein koronares MRT-Bild!) „ausreichen“, die zudem nicht einmal adäquat beschriftet sind.

Auch der klinische Teil weist Miss-, um nicht zu sagen Unverständliches auf. Ein Beispiel: Seit mehr als zwei Jahrzehnten ist die CT als bildgebendes Verfahren der Wahl in der Diagnostik der chronischen Rhinosinusitis etabliert. Im Buchbeitrag auf S. 125 wird jedoch der Eindruck vermittelt, das Krankheitsbild lasse sich bildgebend primär anhand von MRT-Bildern diagnostizieren. Auch wenn sich im Text der Hinweis findet, dass vor einer OP die CT erforderlich ist, wird der unbedarfte Leser hier zunächst auf die falsche Fährte geführt und gewinnt

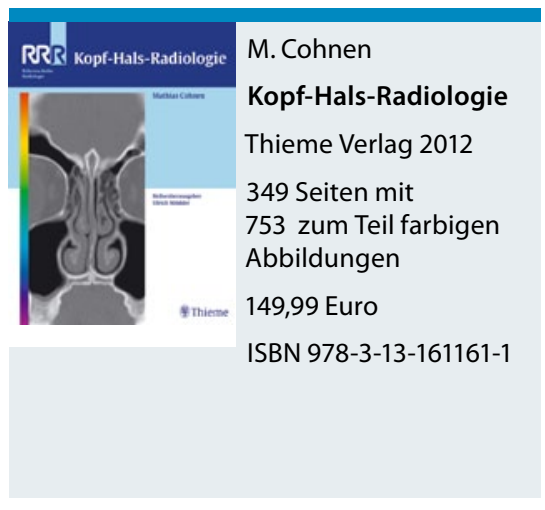

den Eindruck, dass zur Diagnosesicherung der chronischen Rhinosinusitis die MRT obligat ist. Hier rächt sich, dass das Buch aus rein radiologischer Feder entstanden ist, ein HNO-Arzt als Koautor hätte einigen Kapiteln gut getan.

Zusammenfassend haben die Autoren hier ein Werk vorgelegt, dessen Bildmaterial in weiten Teilen durchaus überzeugt; aufgrund der genannten inhaltlichen Schwächen kann es dem HNO-Arzt allerdings nur bedingt empfohlen werden.

Prof. Dr. Gerhard Grevers 